\title{
Recent research activities and results of the Bochum/Bonn Polarized Target Group
}

\author{
Gerhard Reicherz* \\ Ruhr -Universität Bochum, D-44780 Bochum, Germany \\ E-mail: gerhard.reicherzdrub.de
}

\begin{abstract}
The Bochum/Bonn polarized target group is involved in the fixed target experiments COMPASS at CERN and in baryon spectroscopy at ELSA and MAMI. The experiments at Bonn and Mainz are focused on the study of the nucleon resonance region with polarized beam and target with a $4 \pi$ detection system. The focus of the COMPASS experiment is the study of hadron structure and hadron spectroscopy with high intensive muon and hadron beams.

In this proceeding technical achievements and operation experience of the Bonn Frozen Spin Target will be highlighted. New developments to produce an internal superconducting polarizing coil for continuous DNP will be shown. In addition the setup and measurements with the new Bochum NMR system will be presented.
\end{abstract}

XVth International Workshop on Polarized Sources, Targets, and Polarimetry,

September 9-13, 2013

Charlottesville, Virginia, USA

\footnotetext{
* Speaker.
} 


\section{Introduction}

The Standard Model of the strong, electromagnetic and weak interaction is in general very well established. Until recently the Standard Model still had two open ends. On the one side it was the search for the Higgs Boson at highest energies and on the other side it is the formation and understanding of the different forms of strongly interacting matter. First evidence for the possible existence of the Higgs boson was recently reported by the LHC experiments. The Higgs mechanism is responsible for the creation of the masses of quarks and leptons. However, this explains only a few percent of the mass of the nucleons and therefore of the matter surrounding us. The rest of the mass is due to the strong interactions itself. The question how this happens and how one can understand the spectrum and the properties of the emerging strongly interacting particles (hadrons) is the central subject of the CRC $16^{1}$. The aim is to understand the strong interaction in the non-perturbative regime.

\section{Physics programm Bonn CB-ELSA}

The experiments are performed at the electron accelerator ELSA at Bonn. The goal is to gain a good understanding of the spectrum and the properties of baryon resonances and to investigate the underlying QCD dynamics in the photoproduction of mesons. Furthermore, in-medium properties of mesons are investigated. To perform these experiments two detector systems are available, the Crystal Barrel/TAPS and the BGO-OD experiment. The polarized target and the availability of a polarized beam make the measurement of highly sensitive double polarization observables possible. Polarization observables are of decisive importance for an extraction of the resonances from the data. The spectroscopy of baryon resonances ask for double polarization experiments, which are performed to unravel the properties of the pertinent resonances. These data are further investigated in a dedicated multi-channel partial wave analysis together with many other available data sets. The highly complex subject of the CRC 16 can only be advanced by a close cooperation of experiment, theory and partial-wave analysis as well as instrumentation [四].

\section{Polarized target developments}

The frozen spin target is a powerful tool for polarization experiments at low intensity beams but the price for the good acceptance is on one hand a loss in beam time, caused by the need to polarize-up or repolarize the target material, and on another a dedicated railway system to move the detector and external polarizing magnet for the polarization procedure[0].

\subsection{Internal magnet development}

The aim of the project " $4 \pi$-Continuous-Mode-Target" is the combination of the high angular acceptance of the Frozen-Spin-Mode with the high polarization of the Continuous-Mode. For this purpose a cryostat-internal polarizing magnet of the size of the holding coil, as used in the frozen spin mode, is needed. In the following the Bonn development of the "Inversed Notched Coil" concept will be described.

\footnotetext{
${ }^{1}$ Collaborative Research Center 16
} 
For dynamic polarization in the Continuous-Mode a large, external magnet with a flux density of $B=2.5 \mathrm{~T}$ is used. In the target region this has a field homogeneity of $\Delta B / B_{0} \ll e-4$, which is needed to dynamically polarize protons and deuterons. In comparison a solenoid of the size (and thickness) of an internal holding coil has a small total magnetic volume and the ratio to the target volume is small. Such a magnet no longer fulfils the homogeneity conditions and the field must be corrected. In addition, the total thickness of the magnet must be kept small, as the energetic detection threshold of the particles produced in the experiment increases with the thickness of the material.

A manufacturing feasible possibility is the use of correction windings placed on the solenoid ("Notched Coil"'). In can be shown however that to meet the homogeneity condition the tolerance with regards to the positioning in this case is very small. In construction the tolerance criteria for the positioning can not be met due to a lack of guidance of the coil wire. In contrast the correction coils of the "Inversed Notched Coil" are situated within the solenoid. Through two notches in the coil carrier these windings can be optimally positioned, so that winding errors and resulting deterioration of the field homogeneity are minimized.

Such a test coil was constructed on a winding apparatus specifically designed for this purpose. The coil has $2 \times 18$ correction windings with $\sim 3500$ total windings. The use of a multi-filament superconductor $(\varnothing=0.254 \mathrm{~mm}$ ) is needed to create a magnet field of $2.5 \mathrm{~T}$ at a current of $90 \mathrm{~A}$. The total thickness in the region of the correction windings is approximately $2.4 \mathrm{~mm}$, otherwise the thickness is smaller than $2 \mathrm{~mm}$.

A measurement of the critical current at $4.2 \mathrm{~K}$ was successfully carried out. It lies at approximately $71 \mathrm{~A}$ with a central field of $B_{0}=1.9 \mathrm{~T}$. In Fig. $\mathbb{W}$ this point is plotted. For comparison the data supplied by the wire manufacturer is plotted and fitted by the function given by Bottura []]. The projection of this fit to $1 \mathrm{~K}$ gives an estimation that at the working temperature it should be possible to reach a current of $100 \mathrm{~A}$, which is over the required $90 \mathrm{~A}$.

\subsection{Polarization detection}

To determine the absolute value of the target polarization, continuous wave nuclear magnetic resonance (CW-NMR) is the current method of choice. The development of the first Q-meter started 45 years ago. The so called "Liverpool-box", which is still the standard Q-meter, was developed in the early 80s [6]. The "Liverpool-box" is not available any more. The schematics have to be changed, due to the fact that a few circuit components are obsolete. For replacement a new RF-part was developed. The module and its performance will be presented. In other sciences like chemestry or medicine pulsed-NMR is the usual technique.

The advantage of pulsed-NMR is a fast signal detection and a good signal to noise ratio. The measuring time plays a minor role. To determine the polarization only a small amount of the spins should be saturated so that the polarization will be preserved but this reduces the signal to noise ratio. The main motivation to prefer CW-NMR instead of the pulsed-NMR technique is that for the pulsed-NMR after applying the RF-pulse a short part of the FID ${ }^{2}$ - signal is blanked by the ringing of the pulse. During this time (few micro seconds) an important part of quadrupol broadened

\footnotetext{
${ }^{2}$ Free Induction Decay
} 
signal vanishes and a deformed signal results after the Fourier transformation, thus a line shape fit for determination of the polarization is not possible [ [ , Q $]$.

\subsubsection{CW-NMR}

The minimum components of a Q-meter are shown in Fig. \. For the excitation of the spin transition, a RF-signal generator is utilized. For the dynamic nuclear polarization - process (DNP) the magnetic field must be constant, there for the RF-frequency must be varied over the resonance line to detect the whole absorption signal.

The constant current resistor $\mathrm{R}_{c c}$ must be huge compared to the impedance of the series resonance circuit, which consists of a variable capacitor, a dumping resistor $\mathrm{R}_{d}$ and a coaxial transfer cable between the 'warm' part and the induction coil, placed in the cryostat at low temperature in or around the target material. The polarization changes the inductance of the coil with that the quality factor $\mathrm{Q}$ of the resonance circuit changes and this results in a voltage variation which will be amplified and measured with a diode or phase sensitive detector. The integrated signal and therefore the area under the line shape is proportional to the polarization:

$$
P=\frac{2 \hbar I}{g^{2} \mu_{N}^{2} N \pi} \int_{0}^{\infty} \chi(\omega) \frac{\omega_{0}}{\omega} d \omega \equiv \frac{2 \hbar I}{g^{2} \mu_{N}^{2} N \pi} A
$$

As target materials like alcohols, ammonia, carbon hydrogen and lithium-hydrogen are mainly

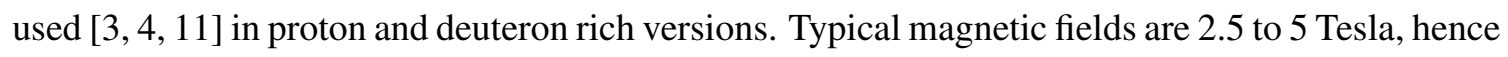
the resonance frequencies are between $10 \mathrm{MHz}$ and $250 \mathrm{MHz}$.

The resonance circuit has to be changeable or should be tuneable in this frequency region. Due to the very small deuteron signal a coil of $\geq 100 \mathrm{nH}$ is applied with a high filling factor. Usually the proton coil consists of one winding with a reduced filling factor. The transfer cable is $n \times \lambda / 2$ or much shorter than $\lambda / 4$ to minimize its influence on the circuit. The capacitor is mechanically or voltage variably tuned to the adequate Larmor frequency. For phase sensitive measurement, in order to increase the signal to noise ratio, the phase cable is varied to zero phase between the two inputs of the phase sensitive detector (Mixer: load and reference signal)[ [8]].

The calibration of the CW-NMR is performed by the $\mathrm{TE}^{3}$-method. The area under the line shape is proportional to the polarization. Therefor by measuring the area units of a signal with a known polarization the proportional factor can be calculated:

$$
P_{d y n}=A U_{d y n} \times \frac{P_{T E}}{A U_{T E}}
$$

The thermal equilibrium polarization for a given magnetic field and temperature can be calculated via the Brillouin function.

For instance the deuteron polarization at a magnetic field of $\mathrm{B}=2.5 \mathrm{~T}$ and a temperature of $\mathrm{T}$ $=1 \mathrm{~K}$ is $P_{T E}=0.052 \%$ (Fig. 田). Maximum polarizations values of $P_{d y n} \geq 80 \%$ are reached [B], 团], corresponding to an enhancement factor $E \geq 80 / 0.052=1538$. The linearity of the Q-meter must be constant over a huge range of a factor of up to $(2000: 1)$.

\footnotetext{
${ }^{3}$ Thermal Equilibrium
} 


\subsubsection{Q-meter}

The RF-part is built of splitters, amplifiers and attenuators on a four-layer circuit board. The tuning box is externally connected via SMA connector to provide a very flexible arrangement. The RF to LF-converter with diode and mixer for a phase sensitive detection was newly designed on a four-layer board. The linearity measurements are performed without dc-offset compensation and further amplification.

To determine the linearity of the Q-meter a reference circuit was adapted and the circuit signal was attenuated in a range of +3 to $-11 \mathrm{~dB}$. In Fig. B the result is presented with a linear fit. The slope had an accuracy of $0.6 \%$ at which the main error results from the measurement of the small excitation voltages [山, ए人].

In Fig. $\boxplus$ shows a thermal equilibrium signal of D-butanol at $1 \mathrm{~K}$ and $2.5 \mathrm{~T}$.

\section{Summary}

The concept of the "Inversed Notched Coil" minimizes the positioning error of the correction coils on the solenoid and fulfils the homogeneity condition that must be obtained to make the polarization of deuterons possible. By using a superconducting wire the thickness of the magnet can be keep very thin. The first prototype built in Bonn fulfills the expectations for the critical current at $4.2 \mathrm{~K}$. A measurement of the field is in progress. Further tests at $1 \mathrm{~K}$ are planned to confirm the estimation of the critical current. A new continuous wave nuclear magnetic resonance Q-meter has been developed to replace the Liverpool-box.

This work is supported by the EU Seventh Framework Programme (HadronPhysics3).

\section{References}

[1] A. Thiel et al. (CBELSA/TAPS Collaboration) Phys. Rev. Lett. 109, 102001 (2012).

[2] C. Bradtke et al.: A New Frozen-Spin Target for $4 \pi$ Particle Detection Nucl. Instrum. and Meth. A 436 (1999) 430-442.

[3] St. Goertz et al.:Polarized H, D and ${ }^{3}$ He Targets for Particle Physics Experiments, Prog. Part.Nucl. Phys. 49 (2002) 403-489

[4] J. Heckmann et al.: Recent Progress in the Nuclear Polarization of Solid Deuterated Butanol Targets, Appl. Magn. Reson. 34 (2008)

[5] L. Bottura: A practical fit for the critical surface of NbT, Applied Superconductivity, IEEE Transactions on 10.1 (2000) 1054Ü1057.

[6] G.R. Court et al.: The development of NMR techniques for the high precision measurement of target polarisation, Nucl. Instr. and Meth. A 324 (1993) 433.

[7] C.M. Dulya: A line-shape analysis for spin-1 NMR signals Nucl. Intr. Meth., A398 (1997) 109-125.

[8] G.R. Court: The development of NMR techniques for the high precision measurement of target polarisation, Nucl. Instr. Meth., A 526 (2004) 65-69.

[9] G. Reicherz et al.: Pulsed NMR for the determination of the nuclear polarization, Nucl. Instr. Meth., A 526 (2004) 96-99 
[10] G. Reicherz: A continuous wave NMR system for detection of the polarization of solid targets, Proceedings PSTP 2011, St. Petersburg (2011) 135-138

[11] C. Hess et al.: Measurement of electron spin-lattice relaxation times in radical doped butanol samples at $1 \mathrm{~K}$ using the NEDOR method, Nucl. Instrum. and Meth., A 694 (2012) 69-77

[12] H. Vondracek: Entwicklung einer HF-Messschaltung zur Polarisationsdetektion, Masterthesis Ruhr-Universitiy Bochum (2013) 


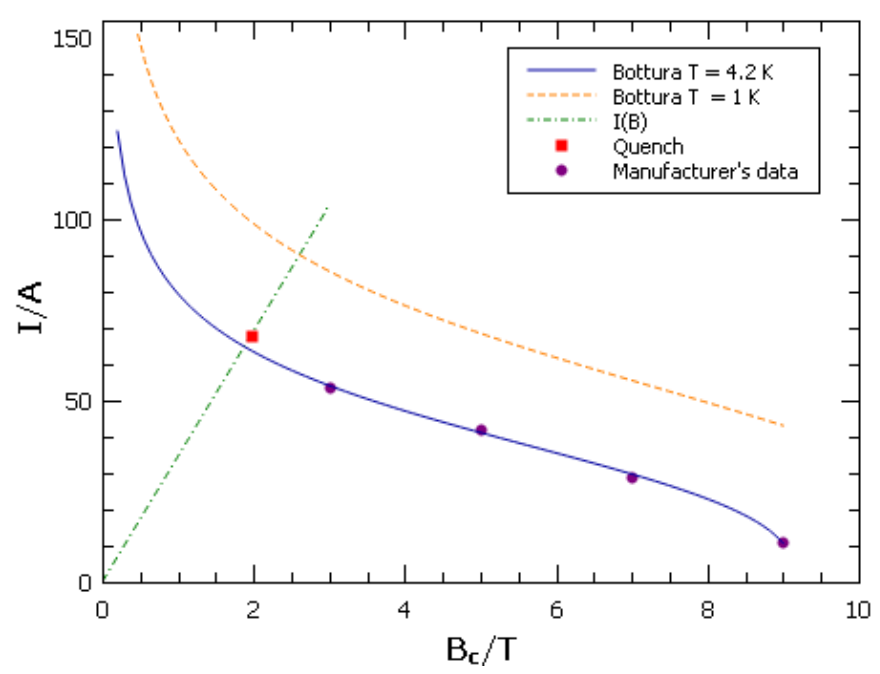

Figure 1: Critical current in dependence of the magnetic field at $4.2 \mathrm{~K}$ and $1 \mathrm{~K}$.

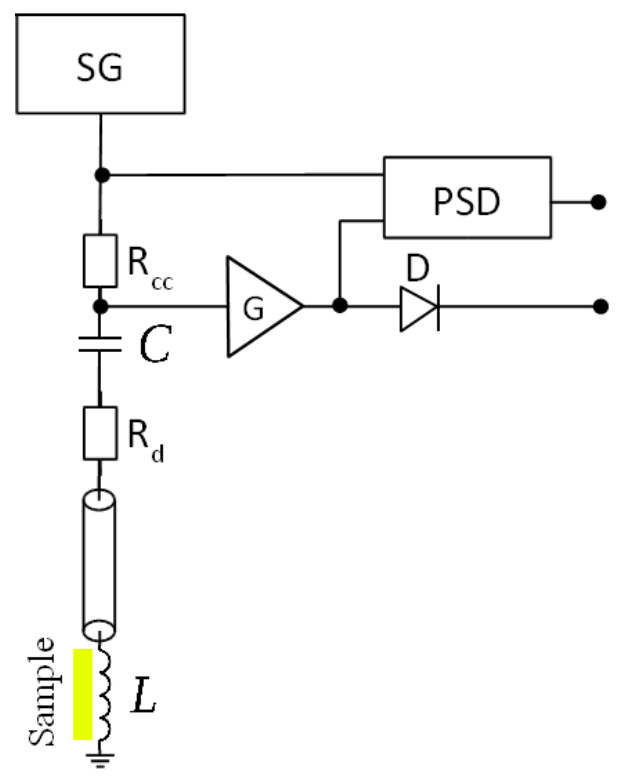

Figure 2: Minimum components of a Q-meter

$\begin{array}{lccc}\text { Nuclei } & \mathrm{B}=2.5 \mathrm{~T} & \mathrm{~B}=3.5 \mathrm{~T} & \mathrm{~B}=5 \mathrm{~T} \\ { }^{2} \mathrm{H} & 16.3 \mathrm{MHz} & 22.8 \mathrm{MHz} & 32.6 \mathrm{MHz} \\ { }^{1} \mathrm{H} & 106.3 \mathrm{MHz} & 148.8 \mathrm{MHz} & 212.6 \mathrm{MHz}\end{array}$

Table 1: Larmor frequencies for protons and deuterons at different magnetic fields 


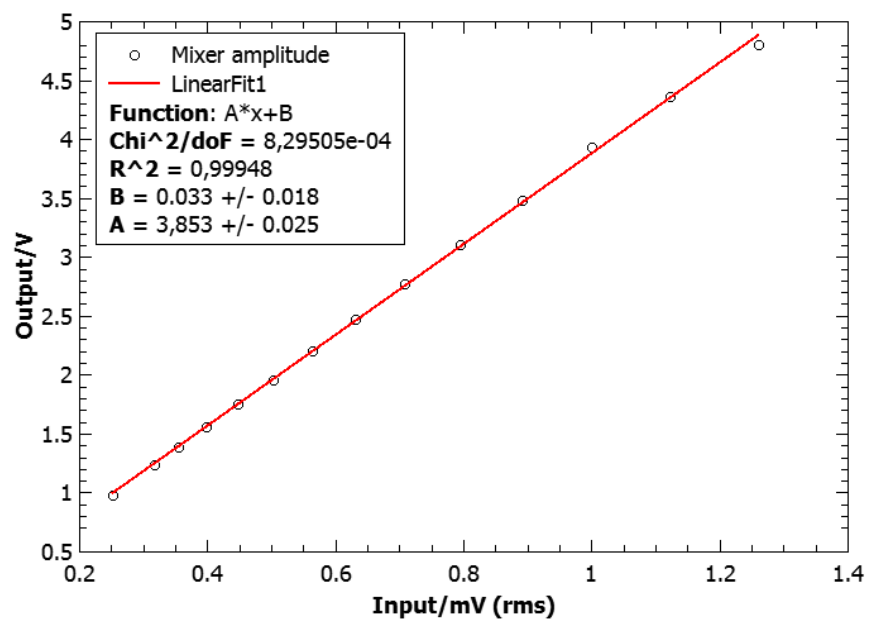

Figure 3: Area units of $\mathrm{NH}_{3}$ TE-signal vs $\mathrm{RF}$ input power from PSD

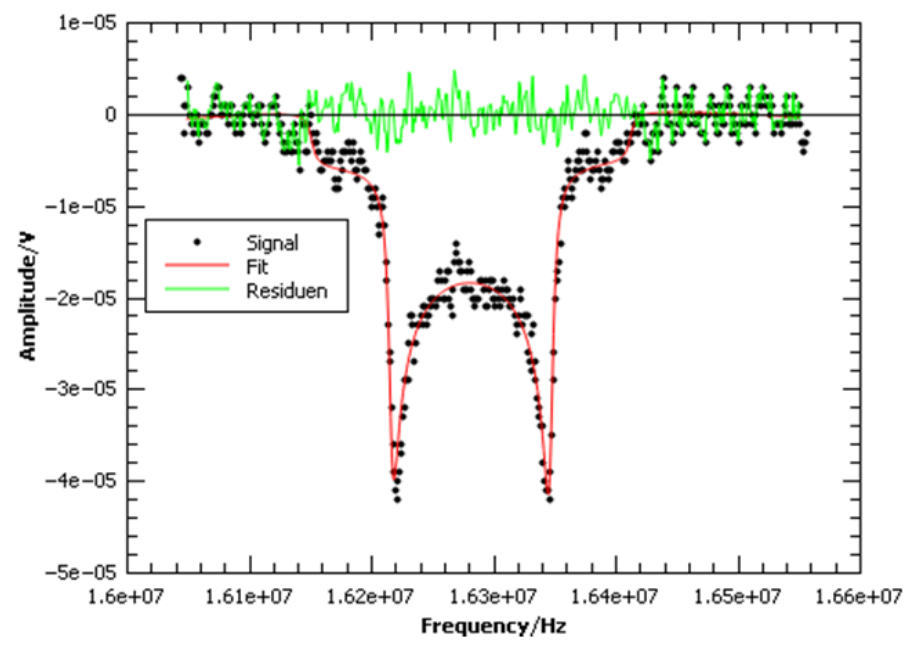

Figure 4: $\mathrm{TE}$ signal of $\mathrm{D}-\mathrm{butanol}$ at $\mathrm{T}=1 \mathrm{~K}$ and $\mathrm{B}=2.5 \mathrm{~T}$ 